\title{
Prototipo de sistema experto basado en lógica difusa para la monitorización del ruido en espacios educativos
}

\section{Prototype of expert system based on fuzzy logic for noise monitoring in educational spaces}

\author{
Aracelis M. González ${ }^{1 *}$, Nahum E. Casco ${ }^{1}$, Robinson H. Melal, Cristian I. Pinzón ${ }^{2}$ \\ ${ }^{l}$ Licenciatura en Ingeniería de Sistemas Computacionales, Centro Regional de Veraguas, \\ Universidad Tecnológica de Panamá, ${ }^{2}$ Facultad de Ingeniería en Sistemas Computacionales, Centro Regional de Veraguas, \\ Universidad Tecnológica de Panamá
}

\begin{abstract}
Resumen Durante los últimos años en Panamá se ha venido incrementado la contaminación acústica, la cual, aunque puede parecer inofensiva, logra alcanzar niveles tan elevados, afectando progresivamente la salud de las personas. Por esta razón, tanto en espacios cerrados, como abiertos es necesario monitorizar los decibeles del ruido. Los sistemas expertos permiten representar conocimientos de expertos, lo cual facilita la toma de decisiones más precisa. Una de las técnicas para la construcción de sistemas expertos es mediante el concepto de lógica difusa. En este artículo se presenta el prototipo de un sistema experto basado en lógica difusa para la monitorización del ruido y un mecanismo de alerta en espacios educativos. El sistema desarrollado plantea la utilización de técnicas de inteligencia artificial para la toma de decisión y mecanismos de salidas que actúan como alertas, concretamente mediante semáforos de estados, indicando el nivel de ruido captado. En este trabajo se presenta la problemática, un prototipo del sistema experto funcional, y los resultados obtenidos de las pruebas realizadas.
\end{abstract}

Palabras clave Contaminación acústica, espacios educativos, inteligencia artificial, sistemas expertos, lógica difusa.

\begin{abstract}
During the last years in Panama, noise pollution has increased, which, although it may seem harmless, manages to reach such high levels, progressively affecting people's health. For this reason, both in closed and open spaces it is necessary to monitor the decibels of the noise. Expert systems enable expert knowledge to be represented, facilitating more accurate decision making. One of the techniques for building expert systems is through the concept of fuzzy logic. This article presents the prototype of an expert system based on fuzzy logic for noise monitoring and an alert mechanism in educational spaces. The developed system proposes the use of artificial intelligence techniques for decision making and exit mechanisms that act as alerts, specifically through state traffic lights, indicating the level of noise captured. This work presents the problem, a prototype of the functional expert system, and the results obtained from the tests carried out.
\end{abstract}

Keywords Acoustic pollution, educational spaces, artificial intelligence, expert systems, fuzzy logic.

*Corresponding author: massielamgb@gmail.com

\section{Introducción}

Diversas instituciones y organizaciones han realizado estudios que demuestran el impacto que generan los altos niveles de ruido. De acuerdo con la Organización Mundial de la Salud (OMS), más de 1000 millones de personas están en riesgo de sufrir problemas auditivos por la exposición a niveles perjudiciales de ruido, donde el $50 \%$ oscila entre jóvenes de 12 a 35 años, con riesgos a los ruidos por aparatos personales y el $40 \%$ a ruidos generados en lugares de ocio [1]. En Panamá las universidades han calculado que alrededor del $70 \%$ de los panameños están expuestos a niveles de ruido superiores al permitido por la OMS [2].

A pesar de los problemas que se generan a partir de la exposición al ruido, se ha demostrado un bajo interés en la búsqueda de soluciones, debido a que los daños que ocasiona no son inmediatos, lo que contribuye a que exista un bajo nivel de concientización y cultura del ruido de la población en general.

En países como España, se han implementado medidas para combatir el ruido desde los centros educativos, optando por 
semáforos de ruido como una forma sencilla, y sobre todo instructiva sobre la importancia de la exposición a niveles altos de ruido. En estos países se han registrado casos de comedores escolares que alcanzan los 100 decibeles (dB), el mismo ruido que ocasiona un tubo de escape, cuando lo ideal es que estos ronden los 60 decibeles y los salones de clases los 40 decibeles [3].

La inteligencia artificial (IA) es una de las áreas de investigación que más desarrollo está teniendo actualmente en diversos campos. Entre las áreas de mayor desarrollo se encuentra la lógica difusa, al tener gran impacto en la solución de problemas reales. La lógica difusa fue planteada por Lotfi Zadeh con el desarrollo de los conjuntos difusos [4], lo que permitió dotar sistemas con la capacidad de evaluar distintos fenómenos con cierto grado de imprecisión, asemejándose al razonamiento natural humano.

Los sistemas expertos son programas inteligentes que permiten replicar el conocimiento de expertos humanos en un dominio específico para la resolución de problemas. Representan el conocimiento, gracias a las técnicas proporcionadas por la IA como la denominada lógica difusa. Esta imita la forma en que un humano toma decisiones muchas veces, con información vaga o imprecisa, y trabaja esos datos mediante un sistema de inferencia basado en reglas "Si... Entonces", que permite trasladar sentencias del lenguaje natural a un lenguaje matemático formal.

El objetivo del presente trabajo es la construcción de un sistema experto basado en el concepto de lógica difusa para la monitorización de la contaminación acústica en centros educativos, y para la toma de decisión de alertar cuando los niveles de ruido pueden ser peligrosos para las personas.

Esta investigación se divide en las siguientes secciones: antecedentes, materiales y métodos, resultados y discusión, y conclusiones.

\section{Antecedentes}

La hipoacusia, mejor conocida como sordera, una de las principales afecciones causadas por el ruido, ya no se ve reflejada solo en adultos expuestos por trabajo o el envejecimiento, sino que también afecta a los jóvenes e infantes en su entorno. En el campo laboral, la pérdida de audición se ha convertido en la principal causa de indemnización, debido a la constante exposición al ruido [5].

En Panamá, en base a estadísticas de la Caja del Seguro Social (CSS), se atendieron para el 2017 un total de 2,300 pacientes con cuadros clínicos de hipoacusia producida por contaminación acústica. Estas atenciones se llevaron a cabo en el laboratorio de audiología del Hospital de Especialidades Pediátricas (HEP), en su mayoría provenientes de las provincias de Panamá, Chiriquí, Coclé y Veraguas [6].
En 2017 se escribió el artículo "Contaminación acústica en el campo de la USMA", en donde se realizó un estudio para medir el ruido en diversas horas del día utilizando sonómetros PCE-322 A y el sistema de posicionamiento global (GPS). Indicando que el campo central de la USMA tiene un ruido tolerable con un promedio anual de $62.35 \mathrm{~dB}$, lo que significa que la USMA está en el rango entre silencioso y ruidoso basado en las recomendaciones de la OMS [7].

Un enfoque distinto, relacionado a la monitorización y alerta, fue el artículo "Diseño e implementación de un sensor para la detección de motosierras de la red inalámbrica de telecomunicaciones para la protección ambiental en el bosque" escrito en el 2005. Es un sistema basado en una red neuronal de una capa entrenada para poder identificar el rango de decibeles generados por una motosierra en el bosque [8].

En comparación con los dos sistemas de monitorización de sonido y el sistema desarrollado, este último se diferencia en la implementación de dos plataformas ampliamente utilizadas para el flujo de datos, reforzando la capacidad de almacenamiento. Utiliza como técnica inteligente al momento de tomar decisiones la lógica difusa, que puede tratar valores imprecisos y darle forma, buscando siempre el estado de clasificación más acertado.

\subsection{Contaminación acústica}

A medida que el incremento y desarrollo humano ha logrado formar grandes concentraciones urbanizadas, los problemas ambientales también han ido en aumento, ya no se considera solo la contaminación del agua y el aire, sino que se introduce un nuevo concepto, la contaminación acústica. Esta abarca los sonidos molestos que perturban el entorno y que al igual que los demás problemas ambientales, pueden ocasionar afecciones a la salud.

En 2008 se crea en Panamá la fundación Oír es Vivir, dirigida por un grupo de Fonoaudiólogas con el fin de atender el ciclo de salud auditiva de niños y adultos. Hace énfasis en la importancia del conocimiento sobre el daño que puede causar el ruido, exponiendo datos como [9]:

La causa principal de pérdida auditiva es la exposición excesiva a los ruidos.

- La exposición continua a ruidos superiores a $85 \mathrm{~dB}$ produce daño de la audición.

- Si el nivel de ruido ambiental impide mantener una conversación, quiere decir que, es excesivo y potencialmente dañino.

- El $25 \%$ de las personas expuestas a ruido excesivo en su lugar de trabajo, desarrollan algún grado de pérdida auditiva.

- Incluso una pérdida auditiva leve puede afectar la capacidad de aprendizaje de un niño. 
La Organización Mundial de la Salud (OMS) plantea una tabla con los decibeles de ruido y sus distintas fuentes [10]. La tabla 1 resume los niveles de ruido, algunas posibles causas y la percepción subjetiva.

Tabla 1. Niveles planteados por la Organización Mundial de la Salud (OMS)

\begin{tabular}{|c|c|c|}
\hline Decibeles & Fuentes & $\begin{array}{c}\text { Percepción } \\
\text { Subjetiva }\end{array}$ \\
\hline 150 & Perforación del tímpano & \multirow{2}{*}{$\begin{array}{c}\text { Intolerable } \\
(110-150)\end{array}$} \\
\hline 110 & Taladrador del pavimento & \\
\hline 80 & Tráfico pesado & $\begin{array}{c}\text { Muy ruidoso } \\
(70-100)\end{array}$ \\
\hline 40 & $\begin{array}{l}\text { Música de radio en tono } \\
\text { bajo }\end{array}$ & $\begin{array}{c}\text { Poco ruido } \\
(30-60)\end{array}$ \\
\hline 10 & Respiración tranquila & $\begin{array}{c}\text { Silencio } \\
(0-20)\end{array}$ \\
\hline
\end{tabular}

Tras evaluar las características del problema estudiado, se plantea la construcción de un prototipo de sistema experto para la monitorización y alerta del ruido en espacios educativos.

\section{Materiales y métodos}

\subsection{Tecnologías implicadas}

Se propone una arquitectura de hardware y software encargados de la captación y procesamiento de los decibeles captados. Para su desarrollo se utilizaron los siguientes materiales:

- Placa Raspberry Pi 3B

- Procesador: Chipset Broadcom BCM2387. 1,2 $\mathrm{GHz}$ de cuatro núcleos ARM Cortex-A53.

- GPU. Dual Core VideoCore IV ${ }^{\circledR}$ Multimedia Co-procesador.

- RAM: 1GB LPDDR2.

- Ethernet socket Ethernet 10/100 BaseT. 802.11

b / g / n LAN inalámbrica y Bluetooth 4.1 (Classic Bluetooth y LE)

- Placa Arduino Mega

- Voltaje Operativo: 5V.

- Voltaje de Entrada: 7-12V.

- Voltaje de Entrada(límites): 6-20V.

- Pines digitales de Entrada/Salida: 54 (de los cuales 15 proveen salida PWM)

- Corriente DC por cada Pin Entrada/Salida: $40 \mathrm{Ma}$.

- Sensor de sonido ky_038

- Voltaje de trabajo: 4-6VDC

- Sensitividad ajustada por potenciómetro

- Señal Digital y Analógica
- capacidad de carga máxima: AC $0 \mathrm{~V}-250 \mathrm{~V} / 10^{\mathrm{a}}$, DC $0 \mathrm{~V}-30 \mathrm{~V} / 10^{\mathrm{a}}$;

- Tiempo de acción: $10 \mathrm{~ms} / 5 \mathrm{~ms}$

- Lámpara de led (verde, amarillo, rojo).

- Voltaje 110-140V.

○ $35 \mathrm{~W}$.

\subsubsection{Software utilizado}

Se utilizaron los siguientes elementos de software encargados del procesamiento de datos.

- Python (3.4)

Se utilizó Python, uno de los lenguajes de programación más poderosos para el análisis de datos [11], para el desarrollo del código en la Raspberry Pi. Esto que permite la inferencia de los decibeles y otorgarle una clasificación.

\section{- Arduino}

Se utilizó Arduino [12], para el desarrollo del código en la placa Arduino Mega, lo que permite controlar el encendido y apagado de las lámparas según la clasificación recibida por la Raspberry Pi y la lectura de decibeles.

\section{- Librerías}

Se utilizaron las siguientes librerías:

- matplotlib.pyplot //Para generar las gráficas de decibeles vs tiempo

- time //Para controlar el tiempo de lectura de datos

- serial //Para la comunicación con la placa Arduino

- $x l w t \quad / /$ Para almacenar los datos captados

- tkinter //Para la creación de la interfaz

- skfuzzy //Para el procesamiento de datos con lógica difusa en base a reglas y gráficas.

\section{- Lógica difusa}

Formalmente, la lógica difusa es una de las ramas de la Inteligencia Artificial que surgió en 1995 [13]. Permite representar el conocimiento para la resolución de problemas. A diferencia de la lógica clásica, es decir, la que toda persona maneja de una forma u otra al momento de tomar decisiones, la lógica difusa no se limita al "blanco o negro" o al "cierto o falso" [14], sino que busca alternativas.

El prototipo emplea el potencial de los sistemas expertos utilizando la lógica difusa como técnica para evaluar los datos (ver figura 1), y advertir sobre los niveles ruido en un área delimitada para que se tomen las medidas correspondientes.

- Relay 
Pinzón (et al): Prototipo de sistema experto basado en lógica difusa para la monitorización del ruido en espacios educativos

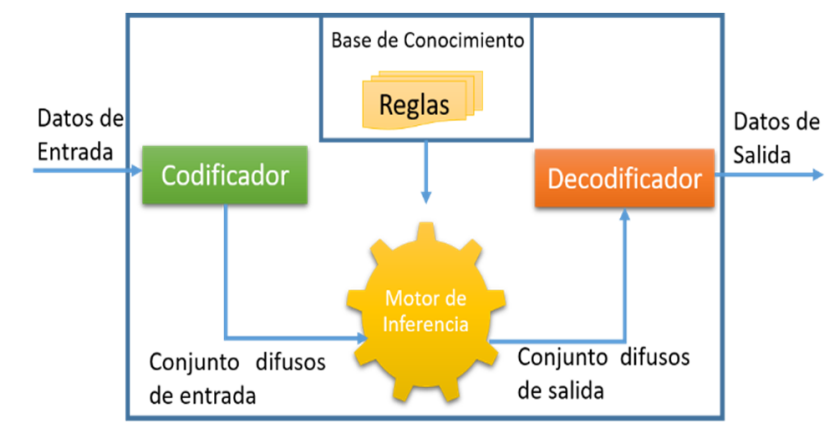

Figura 1. Modelo básico de lógica difusa.

\subsection{Diseño del sistema}

Inicialmente se propone un semáforo que capte los niveles de ruido y los envíe a una placa central para su procesamiento.

A continuación, se presenta un diseño del flujo de datos del semáforo al centro de control del sistema experto basado en una placa Raspberry Pi.

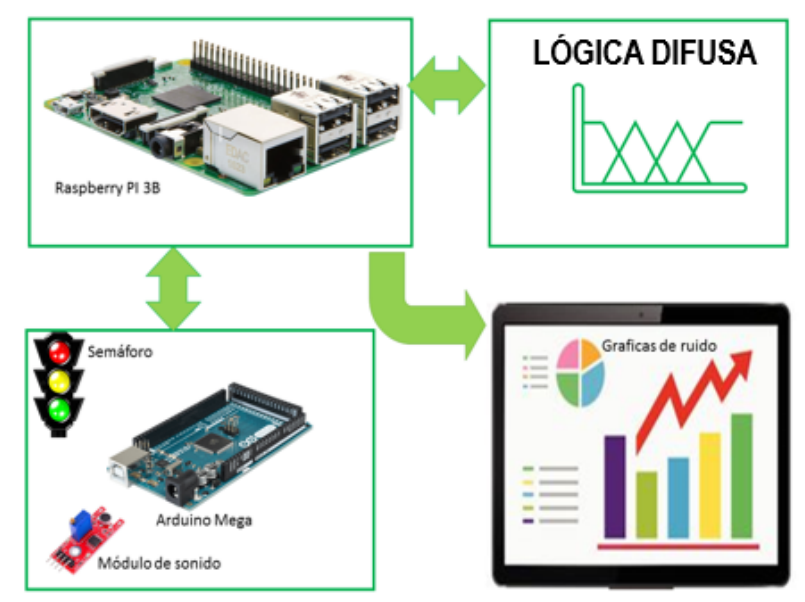

Figura 2. Diseño del sistema experto desarrollado.

Como se aprecia en la figura 2, el semáforo capta los decibeles mediante el sensor de sonido. Los datos son recibidos en tiempo real por la Arduino Mega y transmitidos a la placa central, Raspberry Pi, para ser clasificados mediante reglas de lógica difusa. Una vez son procesados, se devuelve la clasificación al semáforo para representar el estado al que corresponden los niveles de ruido captados. La alerta amarilla refleja un nivel de riesgo, la roja, un nivel que resulta potencialmente peligroso. En cualquier otro caso se mantiene una señal de color verde, indicando que el rango es tolerable en el nivel de decibeles.

\subsection{Población y muestra}

Se realizó una encuesta a diferentes niveles educativos de los distritos de Santiago y Atalaya, provincia de Veraguas, Panamá. Se aplicaron 70 encuestas a nivel primario, 24 a nivel de premedia, 53 a nivel de media y 50 a nivel universitario.

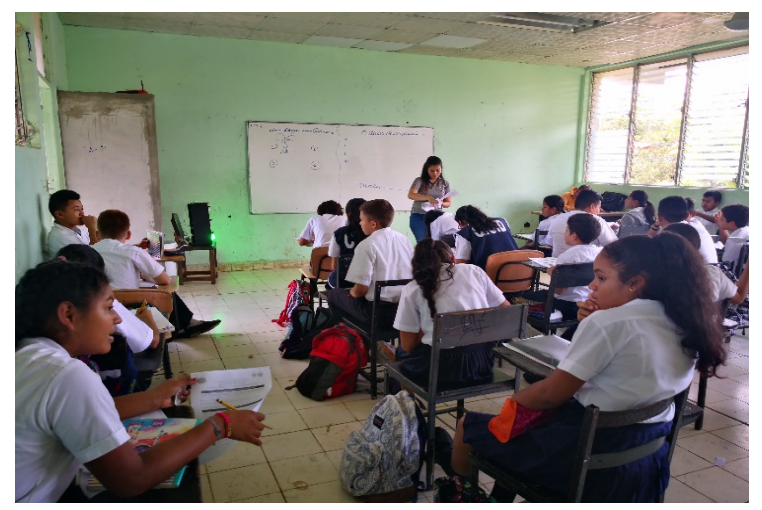

Figura 3. Estudiantes de sexto grado de la Escuela Primaria de Atalaya durante la encuesta.

En total se aplicaron 197 encuestas en los centros educativos:

- Escuela San Antonio

- Escuela San Martín de Porres

- Escuela Primaria de Atalaya (ver figura 3)

- C.E.B.G de Atalaya

- C.E.B.G José Santos Puga

- Instituto Agropecuario Jesús Nazareno

- Instituto Urracá

- Universidad de Panamá, Centro Regional de Veraguas

- Universidad Tecnológica de Panamá, Centro Regional de Veraguas

El $58.3 \%$ de las personas encuestadas demostraron tener conocimiento sobre los problemas que ocasiona el ruido a la salud, mientras que un $41.7 \%$ desconoce sus riesgos (ver gráfica 1). Las personas que consideran que en su colegio los niveles de ruido son altos, equivale al $56.0 \%$, los que consideran que es un espacio libre de ruidos, equivale al $44.0 \%$ (ver gráfica 2). En cuanto al desempeño en clases el $87.0 \%$ considera que el ruido entorpece el aprendizaje, mientras que un $13.0 \%$ considera que no es un factor determinante para el buen desarrollo de la clase (ver gráfica 3). El 20.3\% de las personas consideran que los eventos escolares ruidosos se realizan con mucha frecuencia, en cambio un $79.3 \%$ considera que estos eventos se realizan con poca o ninguna frecuencia.

Por último, considerando la ayuda tecnológica para alertar sobre los niveles de ruido en un salón de clases, el $78.1 \%$ de las personas encuestadas consideran que es útil, mientras que un $21.9 \%$ considera que no se necesita (ver gráfica 4 ).

A continuación, se muestran los resultados de las encuestas graficadas por pregunta con sus respectivas opciones. 
Pinzón (et al): Prototipo de sistema experto basado en lógica difusa para la monitorización del ruido en espacios educativos

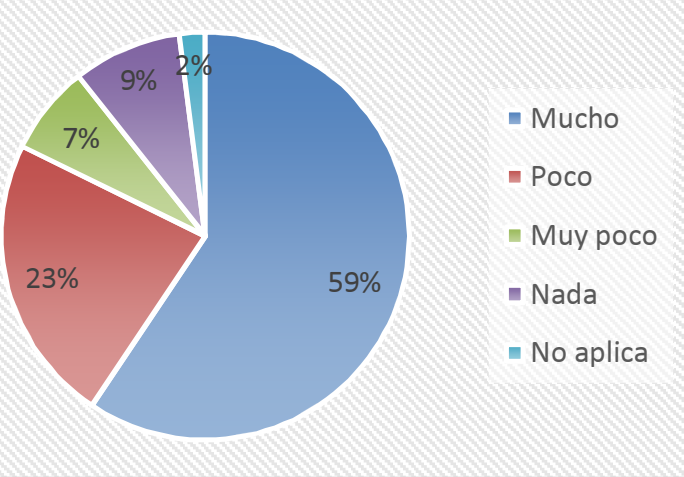

Gráfica 1. ¿En qué medida considera que el ruido afecta la salud?

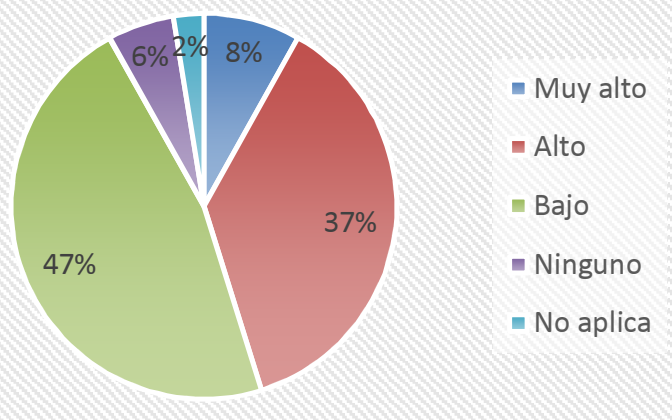

Gráfica 2. ¿Cómo consideras el nivel de ruido en tu colegio?

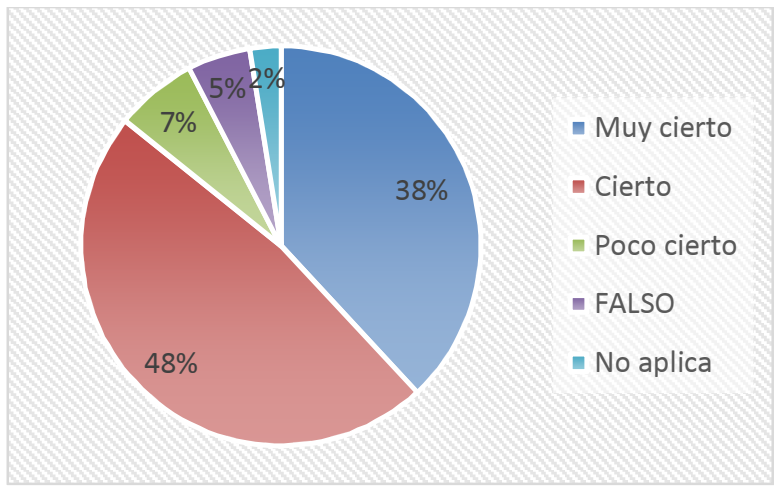

Gráfica 3. ¿Es el ruido un factor que afecta el desempeño en clases?

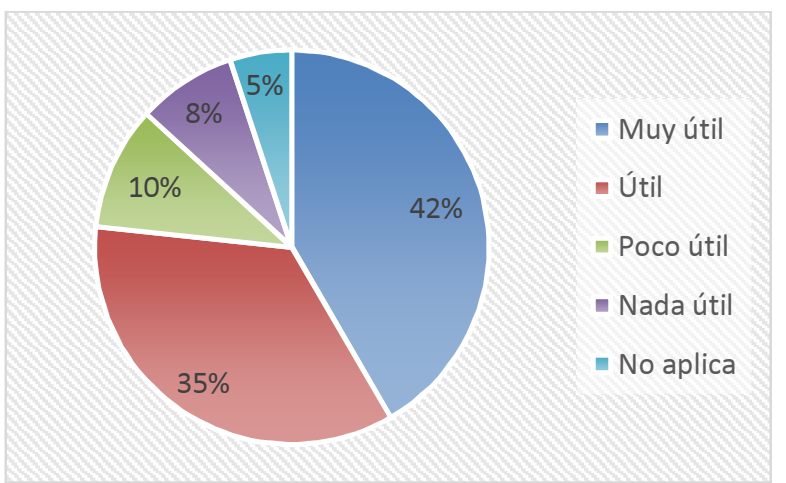

Gráfica 4. ¿Consideras útil un dispositivo tecnológico en tu salón de clases que alerte sobre los niveles de ruido?

\section{Resultados y discusión}

Se visitaron nueve centros educativos de la región, como se listan en el punto 3.3. Con una duración de 10 minutos para la captación de datos en cada caso, se registraron:

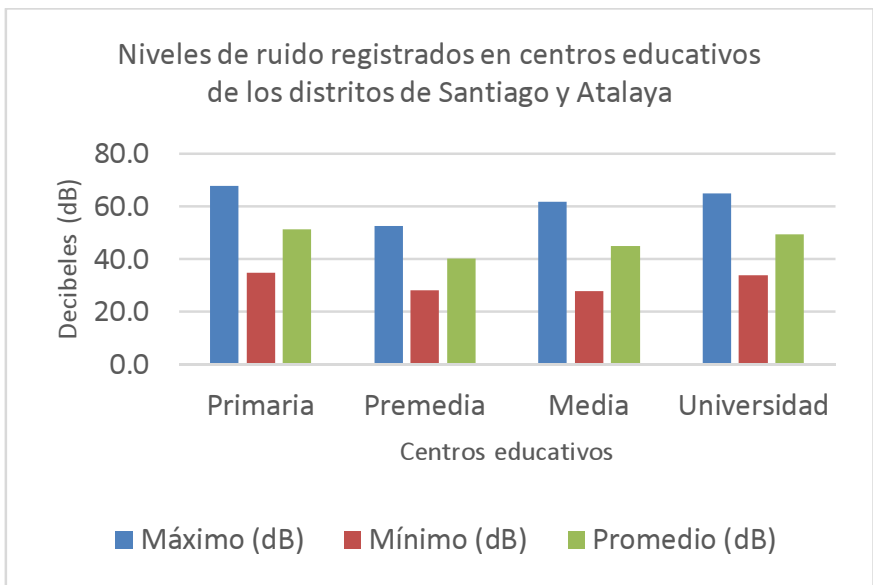

Gráfica 5. Niveles de ruido en centros educativos de los distritos de Santiago y Atalaya.

La grafica 5 refleja que las escuelas primarias y medias registran niveles más altos de ruido que las premedias y universidades, sobrepasando la media de $40 \mathrm{~dB}$ correspondientes a un salón de clases. Esto indica que los estudiantes pueden presentar mayores complicaciones al momento de atender una clase.

Una vez finaliza el intervalo de captación de datos, se calcula un promedio de los valores registrados. Mediante las reglas de lógica difusa se clasifica el valor promedio, dando como resultado la asignación de un estado comprendido en verde, amarillo o rojo, como se muestra la figura 4.

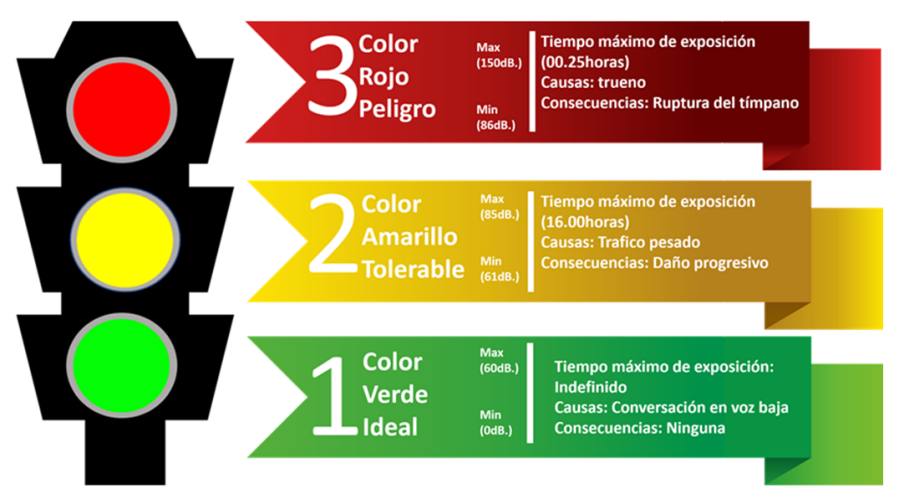

Figura 4. Estados de clasificación del semáforo.

Se realizaron captura de datos en los diferentes centros educativos en horarios matutinos y vespertinos, generando gráficas de decibeles vs tiempo como se muestra en la figura 5. 


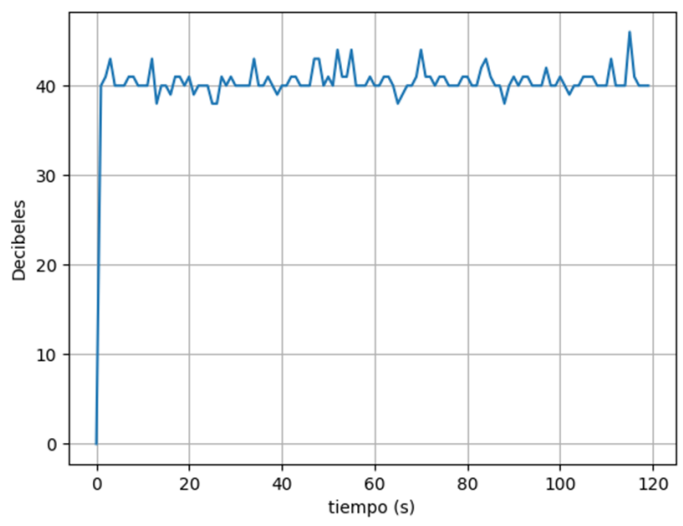

Figura 5. Gráfica de decibeles vs tiempo registrada en la Escuela Primaria de Atalaya.

Mediante el análisis de la gráfica anterior utilizando inferencia difusa, se obtuvo la siguiente clasificación (ver figura 6).

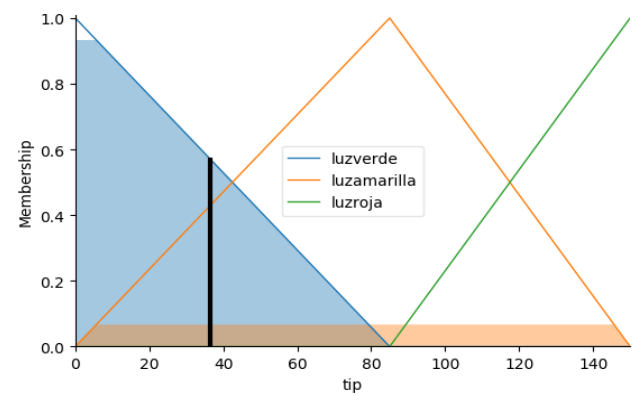

Figura 6. Representación gráfica de los niveles de decibeles captados en un concepto difuso.

La gráfica de la figura 6 se describe como sigue. El color azul (luz verde), amarillo (luz amarilla) y verde (luz roja). Marcó el color azul debido a que los valores captados se encuentran en el rango de 0-60 decibeles, aproximadamente el valor promedio registrado es de 37 decibeles, como demuestra la barra negra. La alerta visual, muestra el estado del semáforo, que advierte mediante el uso del color verde que los niveles del ruido son bajos o tolerables (ver figura 7).

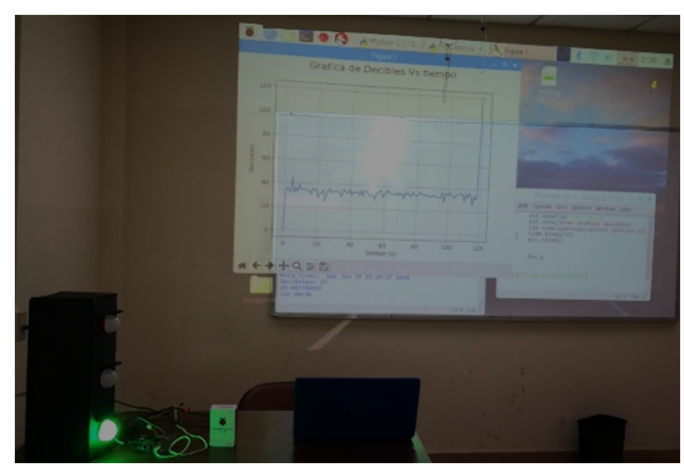

Figura 7. Prototipo funcional del proyecto.
En la figura 7 se muestra la salida del sistema mediante el semáforo que indica un nivel de ruido tolerable que corresponde a los resultados mostrados en la figura 5.

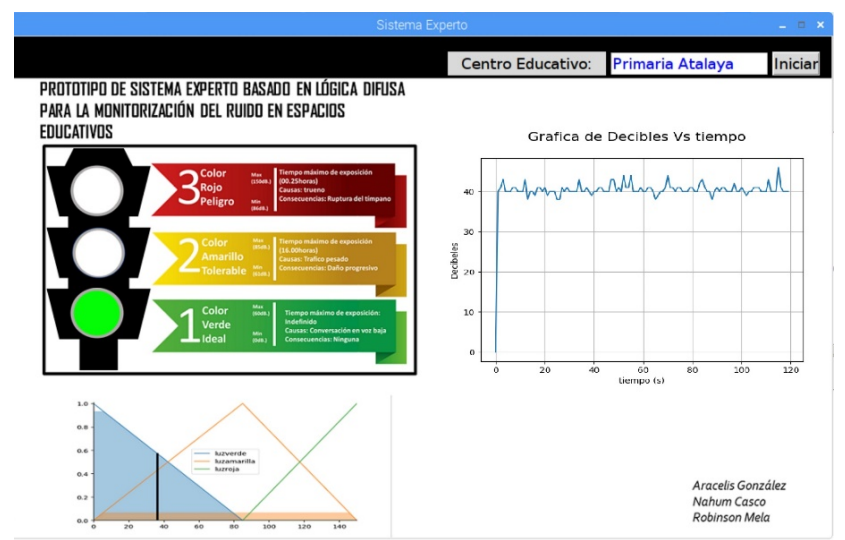

Figura 8. Interfaz del prototipo.

En la figura 8 se muestra la interfaz del sistema, en la cual se aprecian las gráficas de los niveles captados y la clasificación difusa, que deriva en el estado mostrado por el semáforo.

\section{Conclusiones}

En este artículo se ha presentado el diseño de un prototipo de sistema experto basado en lógica difusa para monitorización del ruido en espacios educativos. Los principales logros fueron los siguientes:

Se realizó una encuesta en la cual se logró identificar el grado de desconocimiento de los estudiantes en cuanto a los daños causados por el ruido.

Se hizo el diseño conceptual del sistema para la monitorización del ruido en espacios educativos; de manera flexible para que pueda implementarse en distintas áreas públicas o instituciones educativas.

Se presentó una alternativa para educar a las personas sobre el riesgo ocasionado por el exceso de ruido en espacios públicos.

Se construyó un prototipo que incluye un semáforo de estados que advierte sobre la intensidad del ruido.

\section{AGRADECIMIENTOS}

A la Mgst. María Luisa Vélez por el apoyo y asesoramiento en la formulación de las encuestas.

\section{REFERENCIAS}

[1] "OMS | Escuchar sin riesgos," WHO, 2015.

[2] J. M. Salazar, "Valoración Social del Ruido: Un Problema Urbano," Soc. Rev. Soc. Humanist., vol. Vol. 18, pp. 31-48, 2016

[3] "Educación instalará en todos los comedores escolares vascos 'semáforos de ruido,", El Mundo, Vitoria, 08-Jun-2018. 
[4] S. Russell and P. Norving, Inteligencia Artificial: un enfoque moderno, Segunda Ed. Madrid: PEARSON, 2004.

[5] OMS, "OMS | 10 datos sobre la sordera," OMS. [Online]. Available:

http://www.who.int/features/factfiles/deafness/facts/es/index $2 . h t$ $\mathrm{ml}$

[6] Catherine Perea, "Reportan aumento de evaluaciones para descartar sordera por exceso de ruido," Telemetro, Panamá, 29Apr-2018.[7] G. Burke and F. Lazo De la Vega, "Contaminación acústica en el campus de la Usma", 2017.

[8] W. S. Pérez, "Diseño e implementación de un sensor para la detección de motosierras de la red inalámbrica de telecomunicaciones para la Protección Ambiental en el bosque," Instituto Tecnológico de Costa Rica, 2005.
[9] Fundación Oír es Vivir, "Datos de Interés," Fundación Oír es Vivir, 2017. [Online]. Available: http://www.fundacionoiresvivir.org/quienes-somos/

[10] WHO, “GUÍAS PARA EL RUIDO URBANO,” 1999.

[11] "What's New in Python 2.7 - Python 2.7.15 documentation," Python. [Online]. Available: https://docs.python.org/2/whatsnew/2.7.html

[12] "Introduction Guide," Arduino. .

[13] L. Ballester and A. J. Colom, "Lógica Difusa : Una Nueva Epistemología para las Ciencias de la Educación,” pp. 995-1008.

[14] G. Morales Luna, Introducción a la Lógica Difusa. 2002. 